\title{
Installation of high-valence tungsten in MIL-125(Ti) for boosted photocatalytic hydrogen evolution
}

CrossMark $\leftarrow$ click for updates

\author{
Yijun Zhang ${ }^{1}$, Fangxin $\mathrm{Mao}^{1}$, Yuanwei Liu ${ }^{1}$, Xuefeng Wu${ }^{1}$, Chunfang Wen ${ }^{1}$, Sheng Dai ${ }^{2}$, Pengfei Liu ${ }^{1 *}$ and \\ Huagui Yang ${ }^{1 *}$
}

\begin{abstract}
Metal-organic frameworks (MOFs) possess the features of highly porosity-tunable and electronic-tunable structures, . Taking advantages of these merits, we successfully installed high-valence $\mathrm{W}^{6+}$ ions onto the Ti-oxo clusters of MIL-125(Ti) (W-MIL-125). The installed $\mathrm{W}^{6+}$ ions which form a $\mathrm{W}-\mathrm{O}-\mathrm{Ti}$ structure trigger the metal-to-cluster charge transfer (MCCT), together with an enhanced light absorption. Structural and spectroscopic characterizations reveal that the MCCT process optimizes the charge transfer process and efficiently separates the photogenerated electron-hole spatially. The as-obtained sample of 3.45 W-MIL-125 with optimized electronic structure demonstrates an enhanced photocatalytic hydrogen evolution performance of $1110.7 \pm 63.7 \mu \mathrm{mol} \mathrm{g}^{-1} \mathrm{~h}^{-1}$ under light irradiation, which is $\mathbf{4 . 0}$ times that of the pristine MIL-125(Ti). This work will open up a new avenue for local structural modification of MOFs to boost photocatalytic performance.
\end{abstract}

Keywords: high-valence state, metal-organic frameworks, metalto-cluster charge transfer, photocatalytic hydrogen production

\section{INTRODUCTION}

Photocatalytic hydrogen production by water-splitting has the great potential to alleviate the global energy and environment crisis, and features the advantage in solar energy storage [1-6], which has attracted widespread attention [7-10]. Unfortunately, the unsatisfactory conversion rate of the solar energy to chemical energy restricts the industrial utilization of the photocatalytic technology [11-14]. Therefore, countless efforts have been dedicated to removing the limitation of industrialization with several influential achievements acquired [15-20]. Metalorganic frameworks (MOFs) featuring porous structure and tunable framework, which are liable to modify precisely and explore the catalytic mechanism, have played an increasingly important role in the field of catalysis [21-26]. In recent years, increasing number of attempts have been made to apply MOFsbased materials in the photocatalytic hydrogen production [2731].

In order to expand the application of MOFs-based materials, several novel MOFs-based materials with favorable structure, such as UiO-66 and MIL-125 for the photocatalytic water splitting, have been explored [32-34]. In the previous reports, several strategies like loading co-catalysts [35-37], forming heterojunctions [38-40] and facet engineering [41], have been implemented on the MOFs-based materials, which endows them with a great enhancement in catalytic activity. In addition, it is also effective to modify the local structure of MOFs-based materials, which will lead to an optimized charge transfer process $[42,43]$. Benefited from the tunable structure of MOFs, precise installation of metal ions onto the clusters has been proved to be a successful method to provide active sites for various catalytic reactions with enhanced activity and selectivity $[44,45]$. Such structure optimization strategy also yields a metalto-cluster charge transfer (MCCT), which enables the enhanced spatial electron-hole separation and simultaneously inhibits the charge recombination $[46,47]$. With this in mind, we anticipate this approach can be applied to the reaction of photocatalysis via attaching high-valence heteroatoms onto the MOFs, which were identified as effective promoters in electrocatalysis due to strong electronic interaction [48]. However, limited studies of this strategy have been devoted to improving photocatalytic hydrogen production of MOFs-based materials.

In this work, we have developed a one-step doping strategy of high-valence atoms on Ti-based MOFs MIL-125 to construct local $\mathrm{W}^{6+}$-modified Ti-oxo clusters without disturbing the formation of crystalline structure of MOFs-based materials. The hole attracting high-valence $\mathrm{W}^{6+}$ ion is combined with the Tioxo cluster, performing as an electron donor in the catalytic system. The obtained structure enables an MCCT process, i.e., the holes transfer from the Ti-oxo cluster to the $\mathrm{W}^{6+}$ sites, realizing an electron-hole spatial separation, which further enhances the light absorption, inhibits the charge recombination, favors the interfacial reaction, and prolongs the life time of photogenerated carriers (illustrated in Fig. 1). The resulting WMIL-125 exhibits a four-times enhanced photocatalytic activity for hydrogen generation under light irradiation.

\section{EXPERIMENTAL SECTION}

\section{Materials and characterization}

For the preparation of W-MIL-125, N,N-dimethylformamide (DMF, Shanghai Macklin Biochemical, 99.5\%), methanol $\left(\mathrm{CH}_{3} \mathrm{OH}\right.$, Shanghai Lingfeng Chemical Reagent, 99.5\%),

\footnotetext{
${ }^{1}$ Key Laboratory for Ultrafine Materials of Ministry of Education, Shanghai Engineering Research Center of Hierarchical Nanomaterials, School of Materials Science and Engineering, East China University of Science and Technology, Shanghai 200237, China

${ }^{2}$ Key Laboratory for Advanced Materials and Feringa Nobel Prize Scientist Joint Research Center, Institute of Fine Chemicals, School of Chemistry and Molecular Engineering, East China University of Science and Technology, Shanghai 200237, China

* Corresponding authors (emails: hgyang@ecust.edu.cn (Yang H); pfliu@ecust.edu.cn (Liu P))
} 


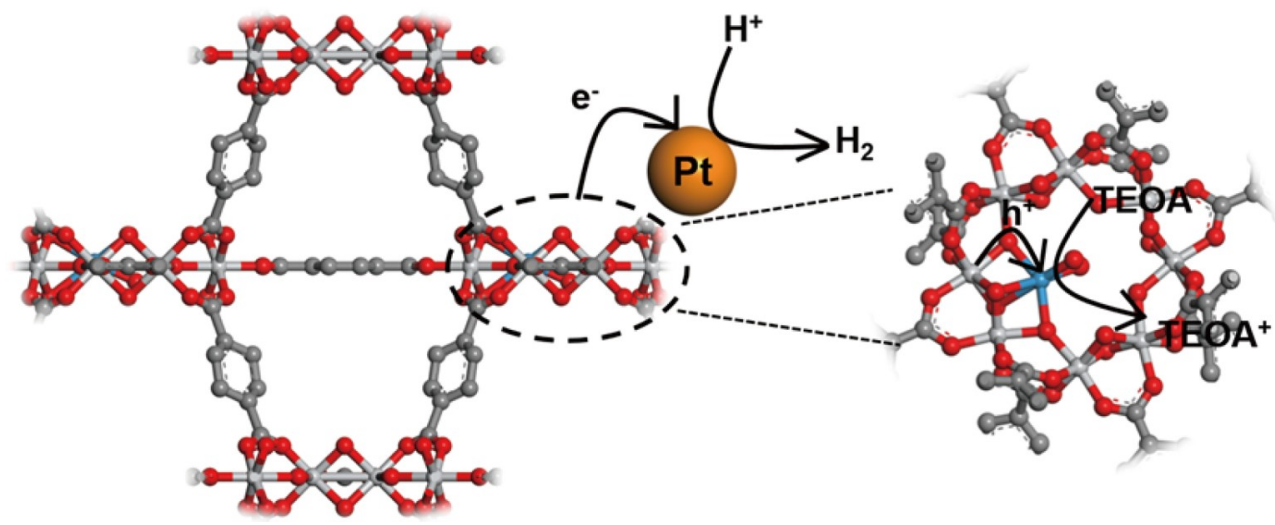

Figure 1 Proposed photocatalytic $\mathrm{H}_{2}$ production mechanism over Pt/W-MIL-125. Color representation: red for O atoms; dark grey for C atoms; light grey for Ti atoms; blue for $\mathrm{W}$ atoms. $\mathrm{H}$ atoms are removed for clarity. The incorporated high-valence $\mathrm{W}$ atom enables an MCCT process, during which the holes transfer from the Ti-oxo cluster to $\mathrm{W}^{6+}$ sites, thus accelerating the electron-hole spatial separation for promoted $\mathrm{H}_{2}$ production performance.

p-phthalic acid $\left(\mathrm{H}_{2} \mathrm{BDC}\right.$, Shanghai Macklin Biochemical, 99.0\%), titanium butoxide $\left(\mathrm{Ti}\left(\mathrm{OC}_{4} \mathrm{H}_{9}\right)_{4}\right.$, Aladdin Industrial, 99.0\%), and sodium tungstate dihydrate $\left(\mathrm{Na}_{2} \mathrm{WO}_{4} \cdot 2 \mathrm{H}_{2} \mathrm{O}\right.$, Sinopharm Chemical Regent, $99.5 \%)$ were used as the ingredients. To evaluate the photocatalytic hydrogen production rate, acetonitrile $\left(\mathrm{CH}_{3} \mathrm{CN}\right.$, Aladdin Industrial, 99.0\%) was used, and trienthanolamine (TEOA, Shanghai Lingfeng chemical regent, 98.0\%) was employed as the sacrificial regent. Chloroplatinic acid $\left(\mathrm{H}_{2} \mathrm{PtCl}_{6}\right.$, Sigma-Aldrich, 99.9\%) was applied for the co-catalyst loading. All the chemicals mentioned in the article were used without further purification. Scanning electron microscopy (SEM) images were acquired by a Japan Hitachi Scanning Electron Microscope (S4800). Transmission electron microscopy (TEM) images were recorded by a high-resolution transmission electron microscope (JEM-2100). High angle annular dark field scanning TEM (HAADF-STEM) images were recorded on a ThermoFisher Themis $\mathrm{Z}$ microscope equipped with two aberration correctors under $300 \mathrm{kV}$ using a convergence semi angle of $11 \mathrm{mrad}$, and inner- and outer collection angles of 59 and $200 \mathrm{mrad}$, respectively. Energy dispersive X-ray spectroscopy (EDS) was carried out using 4 in-column Super-X detectors. The amount of $\mathrm{W}$ element was determined by an American Agilent inductively coupled plasma atomic emission spectrometer (ICPAES) (167-785 nm/725). X-ray diffraction (XRD) patterns were examined by a Japan Rigaku rotating anode X-ray powder diffractometer $(18 \mathrm{KW} / \mathrm{D} / \max 2550 \mathrm{VB} / \mathrm{PC})$. Fourier transform infrared spectroscopy (FTIR) patterns were obtained on an American Nicolet Fourier Transform-Infrared Spectrometer (7800-350 cm $\left.\mathrm{cm}^{-1}, \quad 0.01 \mathrm{~cm}^{-1} / 6700\right)$. Brunauer-Emmett-Teller (BET) specific surface areas were measured by $\mathrm{N}_{2}$ adsorption at $77 \mathrm{~K}$ on a Quantachrome Instruments (Autosorb 1-C). Before the measurement, the samples were dried at $60^{\circ} \mathrm{C}$ under vacuum overnight. The chemical states and valences of the materials were characterized by an American Thermo Fisher X-ray photoelectron spectroscopy (XPS) (ESCALAB 250Xi). The C 1s peak at $284.6 \mathrm{eV}$ was adopted as an internal reference of the samples in this study. X-ray absorption spectroscopy measurements were conducted by the 1W1B beamline of Beijing Synchrotron Radiation Facility. Light absorption measurements were operated by the Lambda 950 ultraviolet-visible-near infrared (UV-Vis-NIR) spectrophotometer. Photoluminescence (PL) measurements were performed on an LS-55 Lumine/ Fluorescence Spectrophotometer. The light with a wavelength of
$250 \mathrm{~nm}\left(\lambda_{\mathrm{ex}}=250 \mathrm{~nm}\right)$ was chosen as excitation source, while a 290-nm-cut filter was utilized to exclude the interference of the light source. The slit width of excitation and emission were set at 10 and $15 \mathrm{~nm}$, respectively. Electro-spin resonance spectrometer (ESR) patterns were obtained on an American Bruker electrospin resonance spectrometer (100 G-18 KG/EMX-8 /2.7).

\section{Preparation of MIL-125(Ti)}

MIL-125(Ti) was synthesized according to the previous report [49]. Generally, $500 \mathrm{mg}$ of $\mathrm{H}_{2} \mathrm{BDC}$ and $0.26 \mathrm{~mL}$ of $\mathrm{Ti}\left(\mathrm{OC}_{4} \mathrm{H}_{9}\right)_{4}$ were dissolved in the mixture of $9 \mathrm{~mL} \mathrm{DMF}$ and $1 \mathrm{~mL} \mathrm{CH} \mathrm{CH}_{3} \mathrm{OH}$. The mixture was stirred for $30 \mathrm{~min}$ to ensure the well dispersion of the solvent. Afterwards, the solution was sealed into a $50-\mathrm{mL}$ Teflon vail and heated at $150^{\circ} \mathrm{C}$ for $20 \mathrm{~h}$. The obtained white powder was collected and washed with $\mathrm{DMF}$ and $\mathrm{CH}_{3} \mathrm{OH}$ twice to remove the redundant reactant. After centrifugation, the product was dried at $60^{\circ} \mathrm{C}$ under vacuum.

\section{Preparation of W-MIL-125(Ti)}

In this work, $\mathrm{Na}_{2} \mathrm{WO}_{4} \cdot 2 \mathrm{H}_{2} \mathrm{O}$ was chosen as the precursor to import $\mathrm{W}$ atoms into the MOF structure. The synthesis procedure is similar to that of the bulk MIL-125(Ti). Typically, $\mathrm{Na}_{2} \mathrm{WO}_{4} \cdot 2 \mathrm{H}_{2} \mathrm{O}$ with different masses $(35.9,18.0$ and $9.0 \mathrm{mg}$ ) was added into the mixture of $\mathrm{H}_{2} \mathrm{BDC}$, DMF, and $\mathrm{CH}_{3} \mathrm{OH}$, before the addition of $\mathrm{Ti}\left(\mathrm{OC}_{4} \mathrm{H}_{9}\right)_{4}$. The as-prepared samples are denoted as 12.5 W-MIL-125, $6.67 \mathrm{~W}$-MIL-125, and $3.45 \mathrm{~W}$ MIL-125, where the numbers $12.5,6.67$ and 3.45 represent the molar ratio of the $\mathrm{W}$ to the metal atom in the solution. (e.g., 3.45 W-MIL-125 means $\left.n_{\mathrm{W}} /\left(n_{\mathrm{W}}+n_{\mathrm{Ti}}\right)=3.45 \%\right)$. The rest of the preparation procedure was totally the same as that of MIL-125 (Ti).

\section{Photocatalytic $\mathrm{H}_{2}$ evolution evaluation}

The photocatalytic $\mathrm{H}_{2}$ production test was performed by a top irradiation type Pyrex cell and an online water splitting evaluation system [33]. In a typical photocatalytic test, the photocatalyst was suspended in the solution of $45 \mathrm{~mL}$ acetonitrile, $1 \mathrm{~mL}$ water, and $5 \mathrm{~mL}$ sacrificial regent TEOA. A $300-\mathrm{W}$ Xe lamp (CEL-HXBF300) was utilized to provide light irradiation with $>300 \mathrm{~nm}$. The amount of the generated gas was measured by an online gas chromatography (Shimadazu 2014C, thermal conductivity detector (TCD), argon as the carrier gas). Before the performance evaluation, $1.5 \mathrm{wt} \%$ platinum was loaded onto 
the catalyst though a photo-deposition method, in which $250 \mu \mathrm{L}$ $\mathrm{H}_{2} \mathrm{PtCl}_{6}$ solution with $1.5 \mathrm{mg}_{\mathrm{Pt}} \mathrm{mL}^{-1}$ was added into the reaction system and irradiated for $2 \mathrm{~h}$ by the same Xe lamp as the activity measurement. To exclude the influence of the $\mathrm{H}_{2}$ generated during the photo-deposition, the gas in the system was eliminated by a vacuum pump.

\section{Photoelectrochemical measurements}

For photocurrent response, a solar light simulator (AM $1.5 \mathrm{G}$ ) with light intensity was used as a light source [50]. $\mathrm{Na}_{2} \mathrm{SO}_{4}$ solution $\left(0.2 \mathrm{~mol} \mathrm{~L}^{-1}\right)$ was used as the electrolyte. The asobtained samples $(2 \mathrm{mg}$ ) were dispersed into the mixture of $1 \mathrm{~mL} \mathrm{CH}{ }_{3} \mathrm{OH}$ and $10 \mu \mathrm{L}$ Nafion. After $30 \mathrm{~min}$ of ultrasonication, $200 \mu \mathrm{L}$ suspension was dropped onto a fluorine-doped tin oxide (FTO) plate to prepare an electrode and dried at room temperature. The measurements were performed at $0.5 \mathrm{~V}$ by an electrochemical workstation (CHI 760E) in a three-electrode system with $\mathrm{Ag} / \mathrm{AgCl} / \mathrm{KCl}$ electrode as the reference electrode and $\mathrm{Pt}$ foil as the counter electrode. For electrochemical impedance spectroscopy (EIS), the characterizations were performed at $-0.3 \mathrm{~V}$ in the frequency range of $10^{-1}$ to $10^{5} \mathrm{~Hz}$. The open circuit potential (OCP) test was conducted under the similar condition of the photocurrent measurement.

\section{RESULTS AND DISCUSSION}

\section{Morphology and crystal structure characterization}

The installation of high-valence $\mathrm{W}^{6+}$ ions onto the MIL-125 structure was accomplished by the one-pot hydrothermal reaction. The obtained samples were labelled according to the molar ratio of $\mathrm{W}$ to all the transition-metal elements in the precursors. The morphology of the obtained MOFs was investigated by SEM. The SEM images indicate that the prepared MIL-125 exhibits a pill-like morphology with the particle size of $\sim 500 \mathrm{~nm}$, which is similar to the reported ones (Fig. S1) [37,49,51]. After the incorporation of $\mathrm{W}$ atoms, the morphology of frameworks was not destroyed, showing the similar pill-like morphology (Fig. 2a). Noteworthily, the particle sizes of the modified MOFs become slightly larger, which is proved by the TEM (Fig. $2 \mathrm{~b}$ and Fig. S1). Furthermore, the pill-like morphology can be observed vertically in the HAADF-STEM image. The corresponding EDS elemental maps of 3.45 W-MIL-125 show the homogenous dispersion of $\mathrm{C}, \mathrm{O}$, and $\mathrm{Ti}$ as well as the imported $\mathrm{W}$ elements (Fig. 2c). The ICP-AES results also confirm the successful incorporation of $\mathrm{W}$ atoms with $n_{\mathrm{W}} /\left(n_{\mathrm{W}}+n_{\mathrm{Ti}}\right)$ molar ratio of $2.43 \%$, which is slightly below the molar fraction of the precursor (3.45\%) (Table S1).

To clarify the crystalline structure of W-MIL-125, XRD was performed (Fig. 3a and Fig. S2). In the XRD patterns, the highvalence $\mathrm{W}^{6+}$ ion does not destruct the MOF crystal structure, and the modified sample exhibits a profile with no obvious difference with the simulated patterns and pristine material. In the FTIR spectra, the pattern of $3.45 \mathrm{~W}$-MIL-125 is in accordance with that of the pristine MIL-125 in the regions of 14001700 and $400-800 \mathrm{~cm}^{-1}$, which belong to the carboxylic acid functional group and $\mathrm{Ti}-\mathrm{O}-\mathrm{Ti}-\mathrm{O}$, respectively (Fig. S3) [49]. The nitrogen adsorption isotherms reveal a gas adsorption of $1122.3 \mathrm{~m}^{3} \mathrm{~g}^{-1}$ for the pristine MIL-125, close to the previous report [37]. A moderate decline in adsorption capacity (e.g., $921.6 \mathrm{~m}^{3} \mathrm{~g}^{-1}$ for $3.45 \mathrm{~W}$-MIL-125) after $\mathrm{W}^{6+}$ modification was observed (Fig. $3 \mathrm{~b}$ and Table S2), together with a decline in the volume of mesoporous structure (Fig. S4), which is possibly ascribed to the blockage of holes by the additional $\mathrm{W}^{6+}$ ions and the enlarged particle sizes $[47,52]$. Therefore, the experimental results verify the introduction of $\mathrm{W}$ dopants into the MIL-125 (Ti) with well-preserved MOF structure.
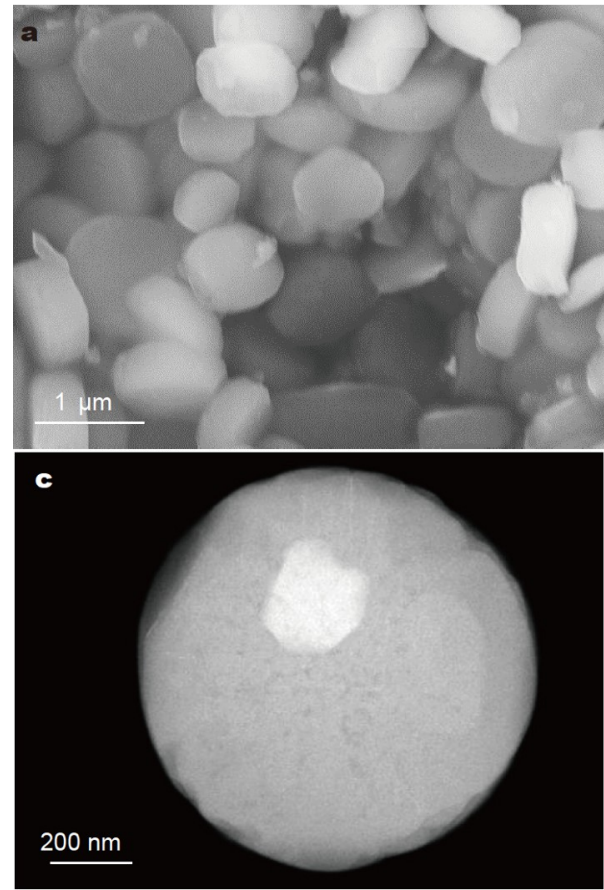

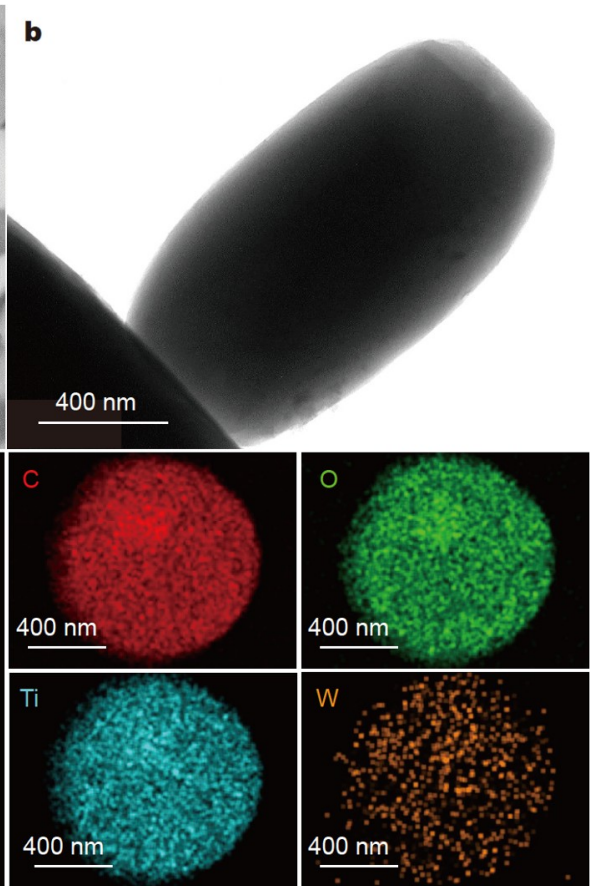

Figure 2 (a) SEM and (b) side-viewed TEM images of the $3.45 \mathrm{~W}$-MIL-125 sample, exhibiting a pill-like morphology, which indicates the $\mathrm{W}^{6+}$ incorporation did not destroy the morphology in the reaction system. (c) Typical vertical-viewed HAADF-STEM image of the 3.45 W-MIL-125 particle and the corresponding elemental maps of $\mathrm{C}, \mathrm{O}, \mathrm{W}$ and $\mathrm{Ti}$, indicative of a homogenous dispersion of $\mathrm{W}$ atoms in the as-obtained sample. 

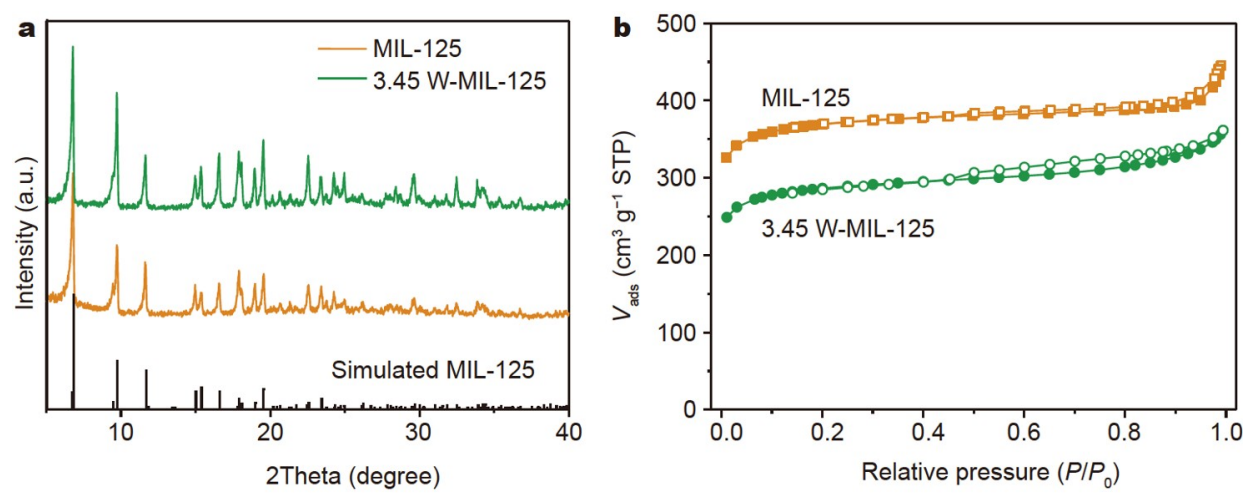

Figure 3 (a) XRD patterns of the pristine and $3.45 \mathrm{~W}$-MIL-125 samples, revealing the unchanged crystalline structure after $\mathrm{W}$ incorporation. (b) $\mathrm{N}_{2}$ adsorption isotherm for the pristine and $3.45 \mathrm{~W}$-MIL-125 materials at $77 \mathrm{~K}$, indicating a specific surface area decline from 1122.3 to $921.6 \mathrm{~m}^{3} \mathrm{~g}^{-1}$ in the adsorption capacity, ascribed to the enlarged particle size and $\mathrm{W}^{6+}$ incorporation.

\section{Valence states and coordination structure analysis}

The surface chemical states of the pristine MIL-125 and $3.45 \mathrm{~W}$ MIL-125 were studied by XPS. The XPS spectrum of Ti $2 p$ in $3.45 \mathrm{~W}$-MIL-125 shows two peaks located at $464.15 \mathrm{eV}\left(\mathrm{Ti} 2 \mathrm{p}_{1 / 2}\right)$ and $458.35 \mathrm{eV}$ ( $\left.\mathrm{Ti} 2 \mathrm{p}_{3 / 2}\right)$, respectively, which belong to the $\mathrm{Ti}^{4+}$ species in the material, displaying negligible discrepancy with the pristine material $(464.15$ and $458.40 \mathrm{eV}$ ) (Fig. S5) [44,53]. As shown in Fig. 4a, no detectable W signals could be observed for the pristine sample. For comparison, obvious $\mathrm{W}$ signals of the $\mathrm{W}^{6+} 4 \mathrm{f}_{7 / 2}$ and $\mathrm{W}^{6+} 4 \mathrm{f}_{5 / 2}$ in the $\mathrm{W} 4 \mathrm{f}$ spectra were detected in 3.45 W-MIL-125. Meanwhile, two peaks centered at 36.60 and $34.45 \mathrm{eV}$ for the spectrum of the reference $\mathrm{Na}_{2} \mathrm{WO}_{4}$ shift to 37.40 and $35.35 \mathrm{eV}$, respectively, which may be due to the influence of the electron-withdrawing capacity of the surrounding Ti-oxo cluster (Fig. S6) [47,54]. Moreover, as shown in Fig. 4 b, the higher peak intensity at $529.80 \mathrm{eV}$ of $3.45 \mathrm{~W}$-MIL125 indicates the higher content of lattice oxygen in the cluster than that of the pristine material, with no oxygen vacancy (Fig. S7) $[53,55]$.

The X-ray absorption near-edge structure (XANES) measurements at $\mathrm{W} \mathrm{L}_{3}$-edge were conducted to analyze the electronic structure of $3.45 \mathrm{~W}$-MIL-125. The white line intensity reveals the distortion of $\mathrm{WO}_{6}$ octahedral symmetry [56]. In Fig. 4c, a higher white line intensity of the $3.45 \mathrm{~W}$-MIL-125 than that of the $\mathrm{WO}_{3}$ reference was observed, suggesting the increased distortion of $\mathrm{WO}_{6}$ octahedral symmetry after incorporation [56]. To obtain more information on the local structure of W-MIL125 , extended X-ray absorption fine structure spectroscopy (EXAFS) was analyzed. In the Fourier-transformed EXAFS (FTEXAFS) spectra (Fig. 4d and Fig. S8), a prominent peak at $1.3 \AA$ (without phase correction if not mentioned) from the $\mathrm{W}-\mathrm{O}$ contribution was detected, which is similar to the $\mathrm{W}-\mathrm{O}$ bond in the $\mathrm{WO}_{3}$ reference. The weaker signal of the $\mathrm{W}-\mathrm{O}-\mathrm{W}$ peak located at $2.6 \AA$ indicates that the majority of the incorporated $\mathrm{W}$ atoms did not form tungsten oxide clusters in $3.45 \mathrm{~W}$-MIL125. Meanwhile, the profile of the sample after modification featuring a distinct peak at $2.4 \AA$ is assigned to the $\mathrm{W}-\mathrm{O}-\mathrm{Ti}$ coordination $[47,57,58]$, which does not exist in the $\mathrm{WO}_{3}$ pattern or $\mathrm{Na}_{2} \mathrm{WO}_{4}$ pattern (Fig. S9). The peak at $2.9 \AA$ may be assigned to the $\mathrm{W} \cdots \mathrm{O} / \mathrm{Ti} / \mathrm{C}$ bonds linked by the oxygen bridge [59]. This result together with the XPS and XANES results confirms the combination of $\mathrm{W}^{6+}$ ions with the Ti-oxo cluster, forming a $\mathrm{W}-\mathrm{O}-\mathrm{Ti}$ structure. Considering the negligible change in the $\mathrm{C}$ 1s spectrum (Fig. S10), it is reasonable to propose that the $\mathrm{W}$ atoms are connected onto the cluster of MIL-125 rather than substituting Ti atoms in the metal cluster.

\section{Photocatalytic performance evaluation}

The optical property of $3.45 \mathrm{~W}$-MIL-125 demonstrates a slightly enhanced light absorption resulting from the modified electronic structure, which was examined by the UV-Vis spectra. According to the pattern, the light absorption of 3.45 W-MIL125 increases in the range of $350-400 \mathrm{~nm}$ (Fig. 5a). The red shift aroused by the $\mathrm{W}^{6+}$-ion incorporation with a certain extension of light absorption narrows the band from $3.70 \mathrm{eV}$ of the pristine material to $3.66 \mathrm{eV}$, estimated by the Tauc plots (Fig. S11). In the band-gap investigation of XPS valence band, 3.45 W-MIL-125 possesses the lower edge of the maximum energy of $2.75 \mathrm{eV}$ than that of the pristine material $(2.87 \mathrm{eV})$ (Fig. S12). Combining with the previous result, it is estimated that the valence band of 3.45 W-MIL-125 is located at $2.90 \mathrm{eV}$, while the conduction band is located at $-0.76 \mathrm{eV}$ [49]. Furthermore, an additional signal was also observed for W-MIL-125 (Fig. S12). Combining with the more gradual decline in the Tauc plots, a mid-gap state estimated at $1.98 \mathrm{eV}$ may be established in W-MIL-125 according to the XPS-valence-band spectra, which offers the theoretical opportunity of hole transfer from the cluster to the high-valence $\mathrm{W}^{6+}$ sites [60]. Meanwhile, the high-valence $\mathrm{W}^{6+}$ sites are affected by the surrounding Ti-oxo cluster, which demonstrates intense electron-withdrawing capacity according to the XPS results. The migration of holes from Ti-oxo clusters to the $\mathrm{W}^{6+}$ sites through an MCCT process can be assumed.

Inspired by the above results, photocatalytic $\mathrm{H}_{2}$ production measurements were set out to evaluate the photocatalytic activity. The experiments were carried out after loading $1.5 \mathrm{wt} \%$ platinum $(\mathrm{Pt})$ though photodeposition by a 300-W Xe lamp $(\lambda>$ $300 \mathrm{~nm}$ ) in the presence of sacrificial regents. As presented in Fig. 5b, when the light irradiation or the photocatalyst was absent, no hydrogen evolution occurred over either the W-MIL125 or MIL-125 samples. When light is on, 3.45 W-MIL-125 shows an enhanced photocatalytic rate of $1110.7 \pm$ $63.7 \mu \mathrm{mol} \mathrm{g}{ }^{-1} \mathrm{~h}^{-1}$, which is four times that of the pristine MIL125 despite the decreased specific surface area. However, the increased amount of additional $\mathrm{W}$ precursors gives rise to an obvious decline in the catalytic activity (Fig. $5 c$ and Fig. S13), which possibly results from the impurity of the as-prepared 

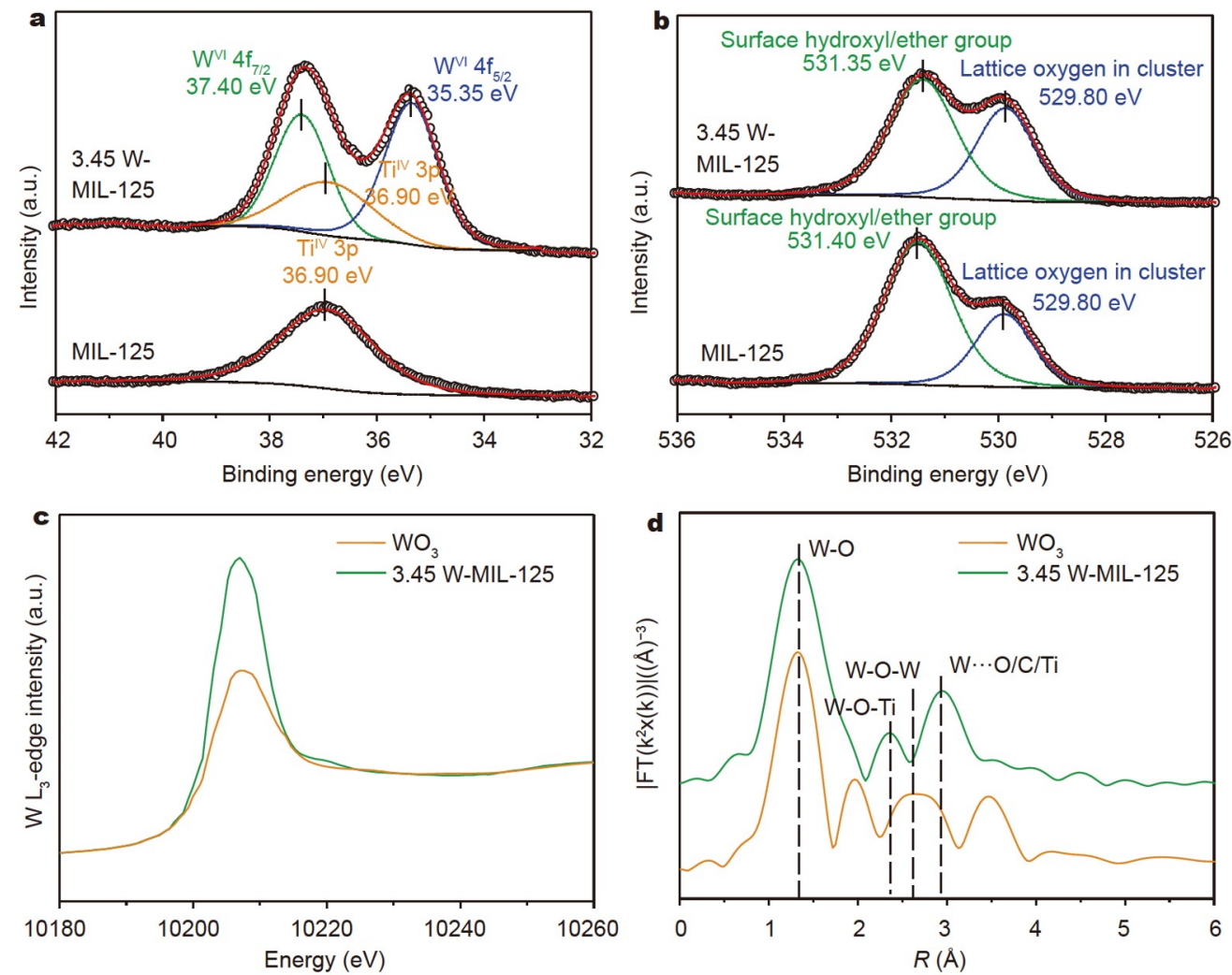

Figure 4 (a, b) XPS spectra of 3.45 W-MIL-125 and the pristine material in the (a) W $4 \mathrm{f}$ and (b) $\mathrm{O}$ 1s regions, respectively, evidencing the successful incorporation of high-valence $\mathrm{W}^{6+}$ ions and the increased amount of lattice oxygen in the cluster. (c) XANES spectra of WO 3 and 3.45 W-MIL-125, showing a higher white line intensity of $3.45 \mathrm{~W}$-MIL-125, which symbolizes an increased distortion of $\mathrm{WO}_{6}$ octahedral symmetry after incorporation into MOF structure. (d) EXAFS patterns of $\mathrm{WO}_{3}$ and $3.45 \mathrm{~W}-\mathrm{MIL}-125$, presenting a weaker intensity in $\mathrm{W}-\mathrm{O}-\mathrm{W}$ and an emerging signal of W-O-Ti.
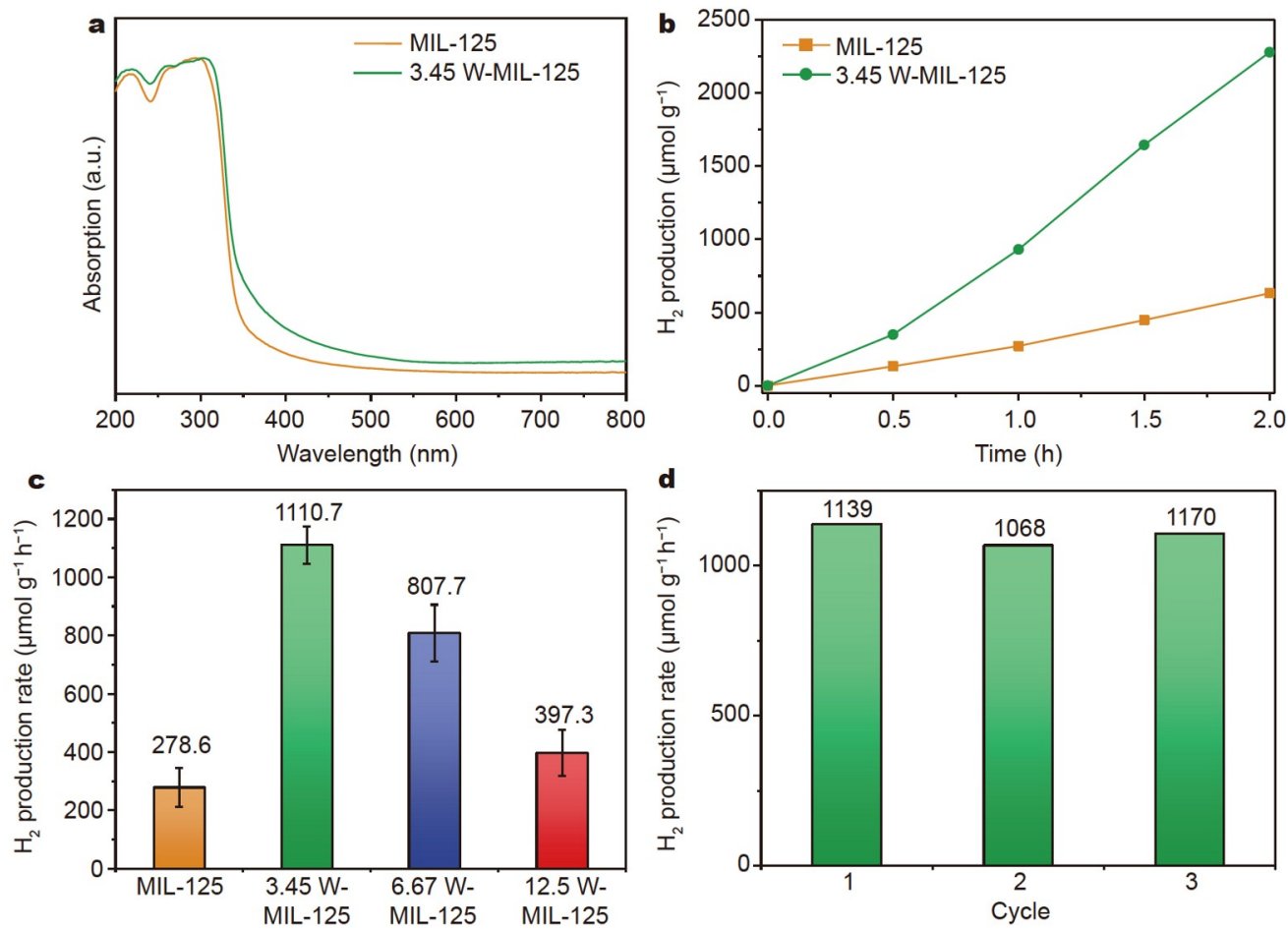

Figure 5 (a) UV-Vis spectra of the pristine and $3.45 \mathrm{~W}$-MIL-125 samples, showing an enhanced light absorption after modification. (b) Photocatalytic hydrogen evolution under light irradiation with sacrificial regent $(\lambda>300 \mathrm{~nm}$, with $1.5 \mathrm{wt} \% \mathrm{Pt})$. (c) Photocatalytic activity difference caused by the amount variation of incorporated W element. (d) Recycling performance of 3.45 W-MIL-125 under light irradiation. 
samples (Fig. S2). In addition, the recycling performance test shows that the hydrogen evolution rate displays negligible deactivation, indicating the good catalytic stability under photocatalytic conditions (Fig. 5d).

The influence of the MCCT process by the incorporation of high-valence $\mathrm{W}^{6+}$ ions and the possible formation of the midgap state in the charge-carrier dynamics were assessed by the PL and photoelectrochemical characterizations. 3.45 W-MIL-125 demonstrates a weaker PL intensity than the pristine material, indicating an improved inhibition of charge carrier recombination (Fig. 6a) $[35,41]$. After the loading of Pt, the data presents a weaker peak than the unsupported catalysts. Furthermore, a similar tendency could be observed between 3.45 W-MIL-125 and the pristine material, which suggests that the advantage of charge separation caused by the high-valence- $\mathrm{W}^{6+}$ incorporation can be maintained after cocatalyst loading. This phenomenon was also supported by the photocurrent measurement, in which the sample after modification possessed an enhancement by about 1.6 times in photocurrent under the irradiation of AM 1.5 $\mathrm{G}$, representing accelerated electron-hole separation (Fig. 6b) $[39,43]$. When the amount of $\mathrm{Na}_{2} \mathrm{WO}_{4}$ increased, the photocurrent was decreased, indicative of an inhibition of electronhole separation. The reduced radius of the semicircle and smaller corresponding charge transfer resistance $\left(R_{\mathrm{ct}}\right)$ in $3.45 \mathrm{~W}$ MIL-125 characterized by the EIS indicate a smaller barrier for interfacial charge transfer than that of the pristine material (Fig. $6 \mathrm{c}$ and Table S3) $[41,43]$. As shown in Fig. 6 d, in the OCP response, $3.45 \mathrm{~W}$-MIL-125 presents a slower decay than the pristine material, which manifests the longer carrier lifetime for the $\mathrm{W}$-incorporated sample [50].

Proposed mechanism for the boosted photocatalytic performance Based on the results listed above, the possible reason for the enhanced performance of 3.45 W-MIL-125 can be concluded. Under light irradiation, the electrons are generated on the $\mathrm{Ti}$ oxo cluster of $3.45 \mathrm{~W}$-MIL-125, simultaneously forming $\mathrm{Ti}^{3+}$ ions according to the previous reports [61], while the holes migrate to the high-valence $\mathrm{W}^{6+}$ sites through an MCCT process, which may be attributed to the mid-gap state formation. Consequently, the photogenerated electrons and holes are accumulated on the Ti-oxo cluster and the incorporated $\mathrm{W}^{6+}$ sites, respectively. The resulting spatial separation of carriers inhibits charge recombination, prolongs the carrier lifetime and accelerates the interfacial charge transfer, leading to an enhanced photocatalytic activity.

\section{CONCLUSIONS}

In summary, through a facile one-step synthesis method, MIL125 structures were successfully established with the importation of high-valence $\mathrm{W}^{6+}$ ions on the Ti-oxo cluster, forming a $\mathrm{W}-\mathrm{O}-$ Ti structure. Owing to the electron-withdrawing capacity of the Ti-oxo cluster, the $\mathrm{W}^{6+}$ sites exhibit a more positive valence state, allowing the holes to transfer from the Ti-oxo cluster to the high-valence $\mathrm{W}^{6+}$ sites. Benefited from the MCCT process, the photogenerated carriers can be spatially separated, allowing efficient charge separation, promoted carrier lifetime and interfacial charge transfer. The as-obtained $3.45 \mathrm{~W}$-MIL-125 demonstrates an enhanced photocatalytic hydrogen production
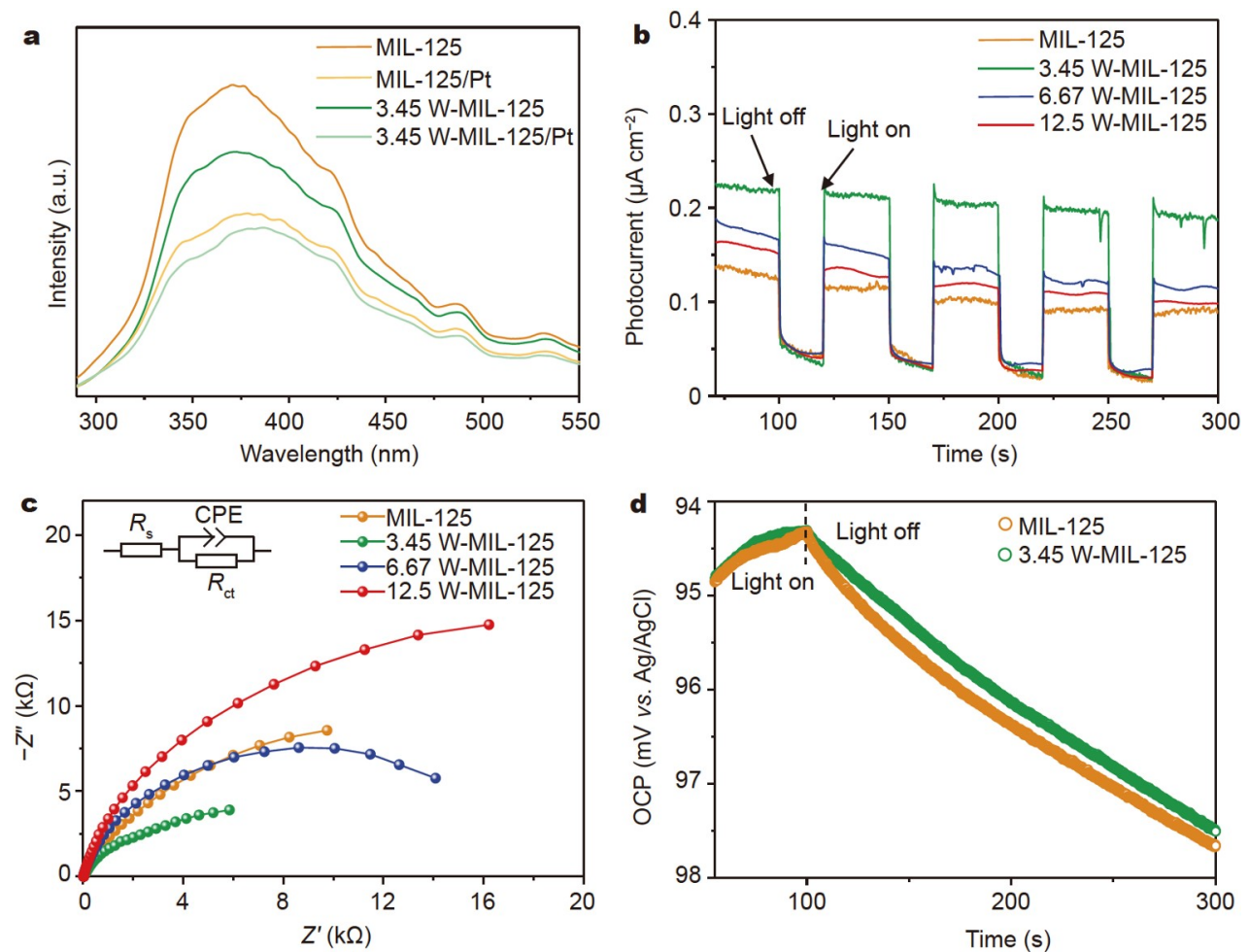

Figure 6 (a) PL spectra of the pristine and 3.45 W-MIL-125 samples with/without Pt decoration, indicative of an inhibited charge recombination after highvalence $\mathrm{W}^{6+}$ incorporation. (b) Photocurrent responses of the samples under AM 1.5 G light irradiation, suggesting a promoted charge separation after $\mathrm{W}^{6+}$ incorporation. (c) EIS spectra of the pristine and $3.45 \mathrm{~W}-\mathrm{MIL}-125$ samples, showing an enhanced interfacial charge transportation. While excess amount of $\mathrm{Na}_{2} \mathrm{WO}_{4}$ was applied in the material, a larger barrier of interfacial charge transfer was detected. (d) OCP results of the as-prepared samples, demonstrating a prolonged lifetime of carriers for $3.45 \mathrm{~W}-\mathrm{MIL}-125$. 
rate of $1110.7 \pm 63.7 \mu \mathrm{mol} \mathrm{g}{ }^{-1} \mathrm{~h}^{-1}$ under light irradiation, which is four times that of the pristine MIL-125(Ti). This work presents an effective strategy of constructing MCCT process by the installation of high-valence heteroatoms, which opens up an avenue for local structural modification of MOFs-based photocatalysts.

\section{Received 12 September 2021; accepted 15 November 2021; published online 17 December 2021}

1 Smith PT, Nichols EM, Cao Z, et al. Hybrid catalysts for artificial photosynthesis: Merging approaches from molecular, materials, and biological catalysis. Acc Chem Res, 2020, 53: 575-587

2 Zhang B, Sun L. Artificial photosynthesis: Opportunities and challenges of molecular catalysts. Chem Soc Rev, 2019, 48: 2216-2264

3 Li A, Zhu W, Li C, et al. Rational design of yolk-shell nanostructures for photocatalysis. Chem Soc Rev, 2019, 48: 1874-1907

4 Wang Y, Vogel A, Sachs M, et al. Current understanding and challenges of solar-driven hydrogen generation using polymeric photocatalysts. Nat Energy, 2019, 4: 746-760

5 Shen R, Ren D, Ding Y, et al. Nanostructured CdS for efficient photocatalytic $\mathrm{H}_{2}$ evolution: A review. Sci China Mater, 2020, 63: 21532188

6 Wang $\mathrm{H}$, Tang Q, Chen Z, et al. Recent advances on silica-based nanostructures in photocatalysis. Sci China Mater, 2020, 63: 2189-2205

7 Zhang H, Lee JS. Hybrid microwave annealing synthesizes highly crystalline nanostructures for (photo)electrocatalytic water splitting. Acc Chem Res, 2019, 52: 3132-3142

8 Chandrasekaran S, Yao L, Deng L, et al. Recent advances in metal sulfides: From controlled fabrication to electrocatalytic, photocatalytic and photoelectrochemical water splitting and beyond. Chem Soc Rev, 2019, 48: 4178-4280

9 Gong J, Li C, Wasielewski MR. Advances in solar energy conversion. Chem Soc Rev, 2019, 48: 1862-1864

10 Tang Y, Zhou $\mathrm{P}$, Wang K, et al. BiOCl/ultrathin polyaniline core/shell nanosheets with a sensitization mechanism for efficient visible-lightdriven photocatalysis. Sci China Mater, 2018, 62: 95-102

11 Yang W, Prabhakar RR, Tan J, et al. Strategies for enhancing the photocurrent, photovoltage, and stability of photoelectrodes for photoelectrochemical water splitting. Chem Soc Rev, 2019, 48: 4979-5015

12 Cheng L, Xiang Q, Liao Y, et al. CdS-based photocatalysts. Energy Environ Sci, 2018, 11: 1362-1391

13 He Y, Hamann T, Wang D. Thin film photoelectrodes for solar water splitting. Chem Soc Rev, 2019, 48: 2182-2215

14 Yin J, Zhan F, Jiao T, et al. Facile preparation of self-assembled MXene@Au@CdS nanocomposite with enhanced photocatalytic hydrogen production activity. Sci China Mater, 2020, 63: 2228-2238

15 Liu J, Liu Y, Liu N, et al. Metal-free efficient photocatalyst for stable visible water splitting via a two-electron pathway. Science, 2015, 347: 970-974

16 Takata T, Jiang J, Sakata Y, et al. Photocatalytic water splitting with a quantum efficiency of almost unity. Nature, 2020, 581: 411-414

17 Wang X, Chen L, Chong SY, et al. Sulfone-containing covalent organic frameworks for photocatalytic hydrogen evolution from water. Nat Chem, 2018, 10: 1180-1189

18 Xiao Y, Feng C, Fu J, et al. Band structure engineering and defect control of $\mathrm{Ta}_{3} \mathrm{~N}_{5}$ for efficient photoelectrochemical water oxidation. Nat Catal, 2020, 3: 932-940

19 Lin L, Lin Z, Zhang J, et al. Molecular-level insights on the reactive facet of carbon nitride single crystals photocatalysing overall water splitting. Nat Catal, 2020, 3: 649-655

20 Hou H, Zeng X, Zhang X. 2D/2D heterostructured photocatalyst: Rational design for energy and environmental applications. Sci China Mater, 2020, 63: 2119-2152

21 Bavykina A, Kolobov N, Khan IS, et al. Metal-organic frameworks in heterogeneous catalysis: Recent progress, new trends, and future perspectives. Chem Rev, 2020, 120: 8468-8535

22 Liu J, Mukherjee S, Wang F, et al. Homochiral metal-organic frame- works for enantioseparation. Chem Soc Rev, 2021, 50: 5706-5745

23 Wei YS, Zhang M, Zou R, et al. Metal-organic framework-based catalysts with single metal sites. Chem Rev, 2020, 120: 12089-12174

24 Tan YX, Wang F, Zhang J. Design and synthesis of multifunctional metal-organic zeolites. Chem Soc Rev, 2018, 47: 2130-2144

25 Chen D, Yang W, Jiao L, et al. Boosting catalysis of Pd nanoparticles in MOFs by pore wall engineering: The roles of electron transfer and adsorption energy. Adv Mater, 2020, 32: 2000041

26 An H, Song J, Wang T, et al. Metal-organic framework disintegrants: Enzyme preparation platforms with boosted activity. Angew Chem Int Ed, 2020, 59: 16764-16769

27 Liang Z, Qu C, Guo W, et al. Pristine metal-organic frameworks and their composites for energy storage and conversion. Adv Mater, 2018, 30: 1702891

28 Wang $\mathrm{H}$, Zhu QL, Zou R, et al. Metal-organic frameworks for energy applications. Chem, 2017, 2: 52-80

29 Xiao JD, Jiang HL. Metal-organic frameworks for photocatalysis and photothermal catalysis. Acc Chem Res, 2019, 52: 356-366

30 Fang $\mathrm{X}$, Shang $\mathrm{Q}$, Wang $\mathrm{Y}$, et al. Single Pt atoms confined into a metalorganic framework for efficient photocatalysis. Adv Mater, 2018, 30: 1705112

31 Sun Y, Lu DF, Sun Y, et al. Large titanium-oxo clusters as precursors to synthesize the single crystals of Ti-MOFs. ACS Mater Lett, 2020, 3: 6468

32 Shi D, Zheng R, Sun MJ, et al. Semiconductive copper(I)-organic frameworks for efficient light-driven hydrogen generation without additional photosensitizers and cocatalysts. Angew Chem Int Ed, 2017, 56: 14637-14641

33 Xiao Y, Qi Y, Wang X, et al. Visible-light-responsive 2D cadmiumorganic framework single crystals with dual functions of water reduction and oxidation. Adv Mater, 2018, 30: 1803401

34 Guo W, Lv H, Chen Z, et al. Self-assembly of polyoxometalates, Pt nanoparticles and metal-organic frameworks into a hybrid material for synergistic hydrogen evolution. J Mater Chem A, 2016, 4: 5952-5957

35 Kampouri S, Nguyen TN, Spodaryk M, et al. Concurrent photocatalytic hydrogen generation and dye degradation using MIL-125- $\mathrm{NH}_{2}$ under visible light irradiation. Adv Funct Mater, 2018, 28: 1806368

36 Sun K, Liu M, Pei J, et al. Incorporating transition-metal phosphides into metal-organic frameworks for enhanced photocatalysis. Angew Chem Int Ed, 2020, 59: 22749-22755

37 Xiao JD, Han L, Luo J, et al. Integration of plasmonic effects and Schottky junctions into metal-organic framework composites: Steering charge flow for enhanced visible-light photocatalysis. Angew Chem Int Ed, 2018, 57: 1103-1107

38 Xu HQ, Yang S, Ma X, et al. Unveiling charge-separation dynamics in $\mathrm{CdS} /$ metal-organic framework composites for enhanced photocatalysis. ACS Catal, 2018, 8: 11615-11621

39 Zhang FM, Sheng JL, Yang ZD, et al. Rational design of MOF/COF hybrid materials for photocatalytic $\mathrm{H}_{2}$ evolution in the presence of sacrificial electron donors. Angew Chem Int Ed, 2018, 57: 12106-12110

40 Peng Y, Zhao M, Chen B, et al. Hybridization of MOFs and COFs: A new strategy for construction of MOF@COF core-shell hybrid materials. Adv Mater, 2018, 30: 1705454

41 Guo F, Guo JH, Wang P, et al. Facet-dependent photocatalytic hydrogen production of metal-organic framework $\mathrm{NH}_{2}$-MIL-125(Ti). Chem Sci, 2019, 10: 4834-4838

42 Zhang J, Bai T, Huang H, et al. Metal-organic-framework-based photocatalysts optimized by spatially separated cocatalysts for overall water splitting. Adv Mater, 2020, 32: 2004747

43 Ma X, Wang L, Zhang Q, et al. Switching on the photocatalysis of metal-organic frameworks by engineering structural defects. Angew Chem Int Ed, 2019, 58: 12175-12179

44 Ji P, Song Y, Drake T, et al. Titanium(III)-oxo clusters in a metalorganic framework support single-site $\mathrm{Co}(\mathrm{II})$-hydride catalysts for arene hydrogenation. J Am Chem Soc, 2018, 140: 433-440

45 Ji P, Manna K, Lin $Z$, et al. Single-site cobalt catalysts at new $\mathrm{Zr}_{12}\left(\mathrm{mu}_{3} \mathrm{O}\right)_{8}\left(\mathrm{mu}_{3}-\mathrm{OH}\right)_{8}\left(\mathrm{mu}_{2}-\mathrm{OH}\right)_{6}$ metal-organic framework nodes for highly active hydrogenation of nitroarenes, nitriles, and isocyanides. J Am Chem Soc, 2017, 139: 7004-7011 
46 Chen X, Xiao S, Wang $\mathrm{H}$, et al. MOFs conferred with transient metal centers for enhanced photocatalytic activity. Angew Chem Int Ed, 2020, 59: 17182-17186

47 Xu C, Pan Y, Wan G, et al. Turning on visible-light photocatalytic C-H oxidation over metal-organic frameworks by introducing metal-tocluster charge transfer. J Am Chem Soc, 2019, 141: 19110-19117

48 Zhang B, Wang L, Cao Z, et al. High-valence metals improve oxygen evolution reaction performance by modulating $3 \mathrm{~d}$ metal oxidation cycle energetics. Nat Catal, 2020, 3: 985-992

49 Han SY, Pan DL, Chen $\mathrm{H}$, et al. A methylthio-functionalized-MOF photocatalyst with high performance for visible-light-driven $\mathrm{H}_{2}$ evolution. Angew Chem Int Ed, 2018, 57: 9864-9869

50 Li D, Yu SH, Jiang HL. From UV to near-infrared light-responsive metal-organic framework composites: Plasmon and upconversion enhanced photocatalysis. Adv Mater, 2018, 30: 1707377

51 Hendon $\mathrm{CH}$, Tiana D, Fontecave $\mathrm{M}$, et al. Engineering the optical response of the titanium-MIL-125 metal-organic framework through ligand functionalization. J Am Chem Soc, 2013, 135: 10942-10945

52 Nasalevich MA, Becker R, Ramos-Fernandez EV, et al. Co@NH $-\mathrm{MIL}-$ 125(Ti): Cobaloxime-derived metal-organic framework-based composite for light-driven $\mathrm{H}_{2}$ production. Energy Environ Sci, 2015, 8: 364375

53 Wang H, Yuan X, Wu Y, et al. In situ synthesis of $\operatorname{In}_{2} \mathrm{~S}_{3} @ M I L-125(\mathrm{Ti})$ core-shell microparticle for the removal of tetracycline from wastewater by integrated adsorption and visible-light-driven photocatalysis. Appl Catal B-Environ, 2016, 186: 19-29

54 Wang $\mathrm{YL}, \mathrm{Nie} \mathrm{T}, \mathrm{Li} \mathrm{YH}$, et al. Black tungsten nitride as a metallic photocatalyst for overall water splitting operable at up to $765 \mathrm{~nm}$. Angew Chem Int Ed, 2017, 56: 7430-7434

55 Zhang B, Zhang J, Tan X, et al. MIL-125- $\mathrm{NH}_{2} @ \mathrm{TiO}_{2}$ core-shell particles produced by a post-solvothermal route for high-performance photocatalytic $\mathrm{H}_{2}$ production. ACS Appl Mater Interfaces, 2018, 10: 1641816423

56 Zhang B, Zheng X, Voznyy O, et al. Homogeneously dispersed multimetal oxygen-evolving catalysts. Science, 2016, 352: 333-337

57 An Y, Liu Y, An P, et al. $\mathrm{Ni}^{\mathrm{II}}$ coordination to an Al-based metal-organic framework made from 2-aminoterephthalate for photocatalytic overall water splitting. Angew Chem Int Ed, 2017, 56: 3036-3040

58 Carrier X, Marceau E, Carabineiro H, et al. EXAFS spectroscopy as a tool to probe metal-support interaction and surface molecular structures in oxide-supported catalysts: Application to $\mathrm{Al}_{2} \mathrm{O}_{3}$-supported $\mathrm{Ni}$ (II) complexes and $\mathrm{ZrO}_{2}$-supported tungstates. Phys Chem Chem Phys, 2009, 11: 7527-7539

59 Sun S, Sun Y, Zhou Y, et al. Shifting oxygen charge towards octahedral metal: A way to promote water oxidation on cobalt spinel oxides. Angew Chem Int Ed, 2019, 58: 6042-6047

60 Liu W, Cao L, Cheng W, et al. Single-site active cobalt-based photocatalyst with a long carrier lifetime for spontaneous overall water splitting. Angew Chem Int Ed, 2017, 56: 9312-9317

61 Chambers MB, Wang X, Ellezam L, et al. Maximizing the photocatalytic activity of metal-organic frameworks with aminated-functionalized linkers: Substoichiometric effects in MIL-125- $\mathrm{NH}_{2}$. J Am Chem Soc, 2017, 139: 8222-8228

Acknowledgements This work was financially supported by the National Natural Science Funds for Distinguished Young Scholars (51725201), the International (Regional) Cooperation and Exchange Projects of the National Natural Science Foundation of China (51920105003), the Innovation Program of Shanghai Municipal Education Commission (E00014), China Postdoctoral Science Foundation Funded Project (2020M681201) and Shanghai Engineering Research Center of Hierarchical Nanomaterials (18DZ2252400). The authors acknowledge the Fundamental Research Funds for the Central Universities (JKD01211519). The authors also thank the Frontiers Science Center for Materiobiology and Dynamic Chemistry, and crew of the 1W1B beamline of the Beijing Synchrotron Radiation Facility for the constructive assistance in the XAFS measurements and data analysis. Dai S acknowledges the support by Shanghai Rising-star Program (20QA1402400) and the Program for Professor of Special Appointment (Eastern Scholar) at Shanghai
Institutions of Higher Learning. Additional support was provided by the Feringa Nobel Prize Scientist Joint Research Center.

Author contributions Liu P and Yang $\mathrm{H}$ designed and supervised the experiments. Zhang Y performed material synthesis, structural characterization and photocatalytic performance evaluation. Mao $\mathrm{F}$ and Wen $\mathrm{C}$ were involved in the data discussion. Liu Y performed XAFS tests and helped with the corresponding data analysis. Wu X and Dai S carried out the HAADFSTEM analysis. All authors were involved in the discussion and writing of this manuscript.

Conflict of interest The authors declare that they have no conflict of interest.

Supplementary information Supporting data are available in the online version of the paper.

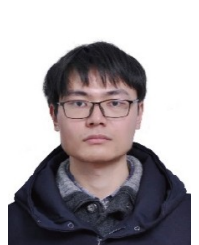

Yijun Zhang is currently a $\mathrm{PhD}$ candidate at East China University of Science and Technology under the supervision of Professor Huagui Yang. His current research interests focus on the design, synthesis and characterization of metal-organic frameworks-based photocatalysts for water splitting.

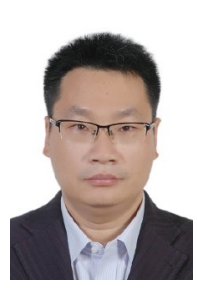

Huagui Yang completed his $\mathrm{PhD}$ degree at the National University of Singapore in 2005. He joined the University of Queensland in 2007 as a postdoctoral research fellow. After finishing his postdoctoral training, he came back to China and took a professor position at East China University of Science and Technology at the end of 2008. His main research interests focus on rational design and fabrication of functional materials for solar-energy conversion.

\section{MIL-125(Ti) 中引入高价态铇元素以促进光催化产氢 性能}

张诣钧 ${ }^{1}$, 毛芳欣 ${ }^{1}$, 刘 袁微 $^{1}$, 吴雪枫 ${ }^{1}$, 温春芳 ${ }^{1}$, 戴 升 $^{2}$, 刘鹏 $^{1^{*}}$, 杨化桂 ${ }^{*}$

摘要 金属有机框架(Metal-organic frameworks, MOFs)材料具有高度 可调控的多孔结构以及电子结构, 在光催化领域非常有前景.本文利用 MOFs的上述特性, 将高价态的铇元素连接在MIL-125(Ti)中的Ti-oxo 团簇上, 得到了 W-MIL-125. 形成的W-O-Ti结构引发了从金属到团簇 的电荷传输(Metal-to-cluster charge transfer, MCCT), 且W-MIL-125具 有更好的吸光性能. 结构和谱学表征证明, MCCT过程能优化电荷的传 输路径, 有效地实现了载流子空间分离. W-MIL-125在光照条件下展现 出更高的产氢性能, 高达 $1110.7 \pm 63.7 \mu \mathrm{mol} \mathrm{g}^{-1} \mathrm{~h}^{-1}$, 是本体样品产氢速 率的 4.0 倍. 本工作为MOFs基材料的局域结构改性以提升光催化性能 提供了新的思路. 